\title{
Oficinas de Planejamento em Saúde na Disciplina de Saúde Coletiva: Relato de Experiência
}

\section{Rafael Esdras Brito Garganta da Silva, Antônio de Pádua Quirino Ramalho², Carla Caroline Oliveira de Souza ${ }^{1}$}

${ }^{1}$ Acadêmicos do curso de Medicina da Universidade Federal do Amazonas

${ }^{2}$ Professor do Departamento de Saúde Coletiva da Universidade Federal do Amazonas

* Autor para correspondência: medresdrasbg@gmail.com

\section{RESUMO}

O conceito de saúde não é somente ausência de doença, mas envolve uma série de fatores condicionantes e determinantes. Considerando essa complexidade, surge a necessidade de ser implementada a saúde coletiva na matriz curricular do curso de Medicina. No interior de suas subáreas estão inseridos o planejamento e a gestão em saúde; a partir dos mesmos, é possível construir estratégias e identificar oportunidades para solucionar problemas, sobretudo na atenção primária. Na disciplina Saúde Coletiva IV do curso de Medicina da Universidade Federal do Amazonas (UFAM), os alunos são imersos em atividades voltadas para o tema, tais como as oficinas de planejamento. No total ocorreram três, e por meio da organização de grupos foi possível discutir sobre prevalência de doenças, situações de risco em saúde e vulnerabilidade social. Experiências como essas devem ser mais frequentes na formação de novos profissionais, para que os mesmos estejam aptos não apenas para o mercado de trabalho, mas para a construção de novas propostas à saúde pública.

Palavras-Chave: Planejamento; Saúde Pública; Educação Médica.

\begin{abstract}
The concept of health is not only the absence of disease, but involves a series of determining factors. Considering this complexity, it is necessary to implement the collective health in the curricular matrix of the Medicine course. Within its subareas are inserted health planning and management, and from them it is possible to build strategies and identify opportunities to solve problems, especially in primary care. In the course Collective Health IV of the UFAM Medicine course, students are immersed in activities focused on the theme, such as planning workshops. In total, there were three of them, and through the organization of groups it was possible to discuss the prevalence of diseases, health risk situations and social vulnerability. Experiences such as these should be more frequent in the training of new professionals, so that they are able not only to the labor market, but in the construction of new proposals for public health.
\end{abstract}

Keywords: Planning; Public Health; Medical Education.

\section{Introdução}

A saúde coletiva é fundamental na formação médica, pois fornece uma visão de saúde abrangente em vários aspectos. Tal área de estudo, segundo Tambellini e Câmara (1998), considera esferas biológicas, sociais, psíquicas e ecológicas, trabalhando assim com o individual e o coletivo. Sob a perspectiva de Carvalho e Ceccim (2006), essa área então nasce como o espaço para discussão da relevância social no processo de formação de profissionais de saúde.
Após uma série de movimentos ligados à reforma sanitária, a saúde coletiva teve seus conceitos mais consolidados e pôde então ampliar, assim como ressingularizar, o campo de atuação dos profissionais de saúde, conforme discutido no estudo de Souza et al. (2017). A prática clínica começou a incorporar uma análise das circunstâncias sociais da vida e da conveniência com fatores determinantes e condicionantes do processo saúde-doença, segundo Rodrigues e David (2015). 
Nesse aspecto, insere-se o processo de planejamento em saúde. Para Paim (2003), o mesmo se refere ao processo de identificação de problemas e oportunidades para serem orientadas ações específicas. Nesse tipo de planejamento, os problemas fazem referência ao estado de saúde, como as doenças, agravos, vulnerabilidade e riscos, e aos serviços de saúde.

Paim (2006) também entende o planejamento como uma ação social, no sentido de ser uma alternativa à improvisação, dando oportunidade de usar a liberdade de um sujeito, individual ou coletivo, a não ser presa fácil dos fatos e circunstâncias. Desse modo, há uma grande importância em trabalhar tal assunto no meio acadêmico. Essa estratégia utilizada demonstrou que os alunos podem ser atores no processo reflexivo diante dos problemas no serviço de saúde.

Com base nisso, a disciplina Saúde Coletiva IV visa a trabalhar nesse ponto. Sua finalidade é capacitar futuros médicos, por meio do ensino, a estarem atentos para as principais necessidades de uma determinada população quanto ao serviço de saúde, estabelecendo estratégias e políticas para serem articuladas com o poder público. Pensando nisso, os alunos de Medicina são estimulados à reflexão e à análise da realidade dos problemas no estado do Amazonas, especificamente. Pretende-se assim detalhar no presente relato de experiência as atividades realizadas nas oficinas de planejamento.

\section{Relato de Experiência}

A disciplina Saúde Coletiva é ministrada em quatro períodos da grade curricular do curso de Medicina da Universidade Federal do Amazonas (UFAM). No quarto período, a disciplina é intitulada Saúde Coletiva IV e aborda temas voltados para o planejamento e gestão em saúde. O objetivo é despertar nos alunos um senso crítico quanto ao tema, para adquirirem novos conhecimentos e discutirem propostas políticas voltadas à promoção da saúde na atenção primária.

A metodologia de ensino empregada na disciplina compreende: aulas teóricas que se utilizam de recursos visuais, discussão de casos e seminários; oficinas de planejamento, que tiveram como objetivo incentivar os alunos a produzirem instrumentos para discussão de problemáticas de saúde pública e aulas práticas que se resumem a visitas a diferentes cenários para discussão de planejamento e situação de saúde de áreas específicas de Manaus.

Na primeira oficina de planificação (Figura 1), o professor dividiu a turma de alunos em grupos e, posteriormente, disponibilizou papéis aos estudantes. Eles continham informações sobre: o número de famílias cadastradas no estado do Amazonas sendo acompanhadas pela atenção primária, segundo dados do Sistema de Atenção Básico (SIAB) proveniente do Departamento de Informática do SUS (DATASUS); outra folha continha estimativa populacional do Amazonas de 2001 a 2014, segundo dados do IBGE (2017); por fim, foi entregue um artigo impresso sobre a prevalência de hipertensão arterial na população brasileira. O intuito da atividade era comparar o número da população acompanhada pelo serviço de saúde com a estimativa total da população hipertensa no estado do Amazonas, e ao final, demonstrar que o estado não realiza uma cobertura adequada no controle de doenças crônicas como a hipertensão.

Outra oficina foi realizada, na qual se discutiu o uso de parâmetros do SUS, e dessa forma foi possível refletir com mais propriedade sobre a análise situacional de saúde (Figura 2). Para isso, consultou-se o documento referente à portaria n. 1.101 de 2002 (BRASIL, 2002). Um dos parâmetros discutidos na aula foi aquele relativo ao número de consultas por habitantes. Considera-se adequado que cada habitante numa população necessita ter pelo menos duas a três consultas médicas por ano. Outro dado importante utilizado na atividade é que há uma estimativa de que 7\% a 9\% da população têm necessidade de internações hospitalares por ano. Consultando essas informações e outras, os alunos foram incentivados a estimar números referentes aos adolescentes em situação de risco de saúde, a prevalência de doenças crônicas na população adulta, idosos em situação de risco de saúde e cobertura do atendimento de saúde bucal. Para isso 


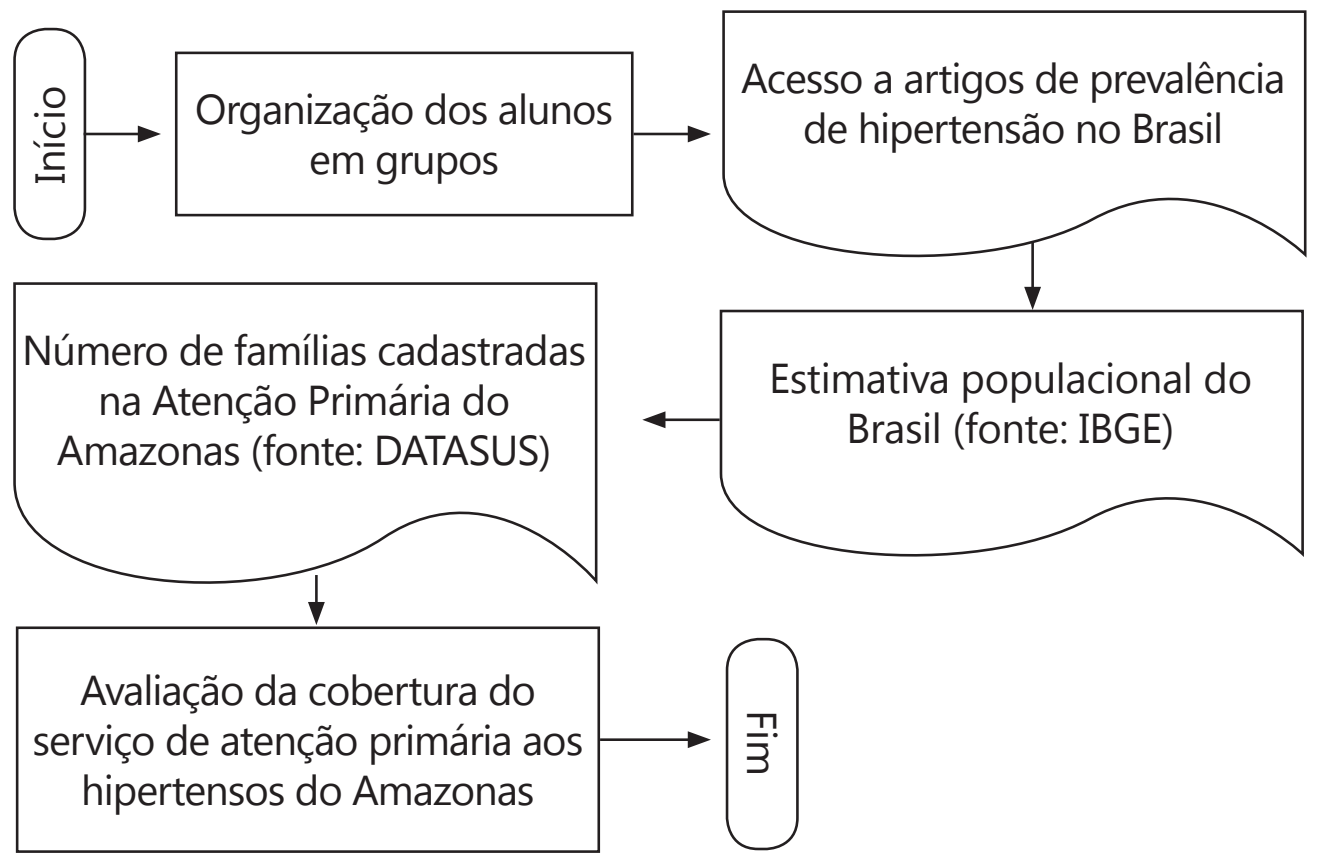

Figura 1 - Fluxograma da oficina I de planejamento. Elaborado por Rafael Esdras.

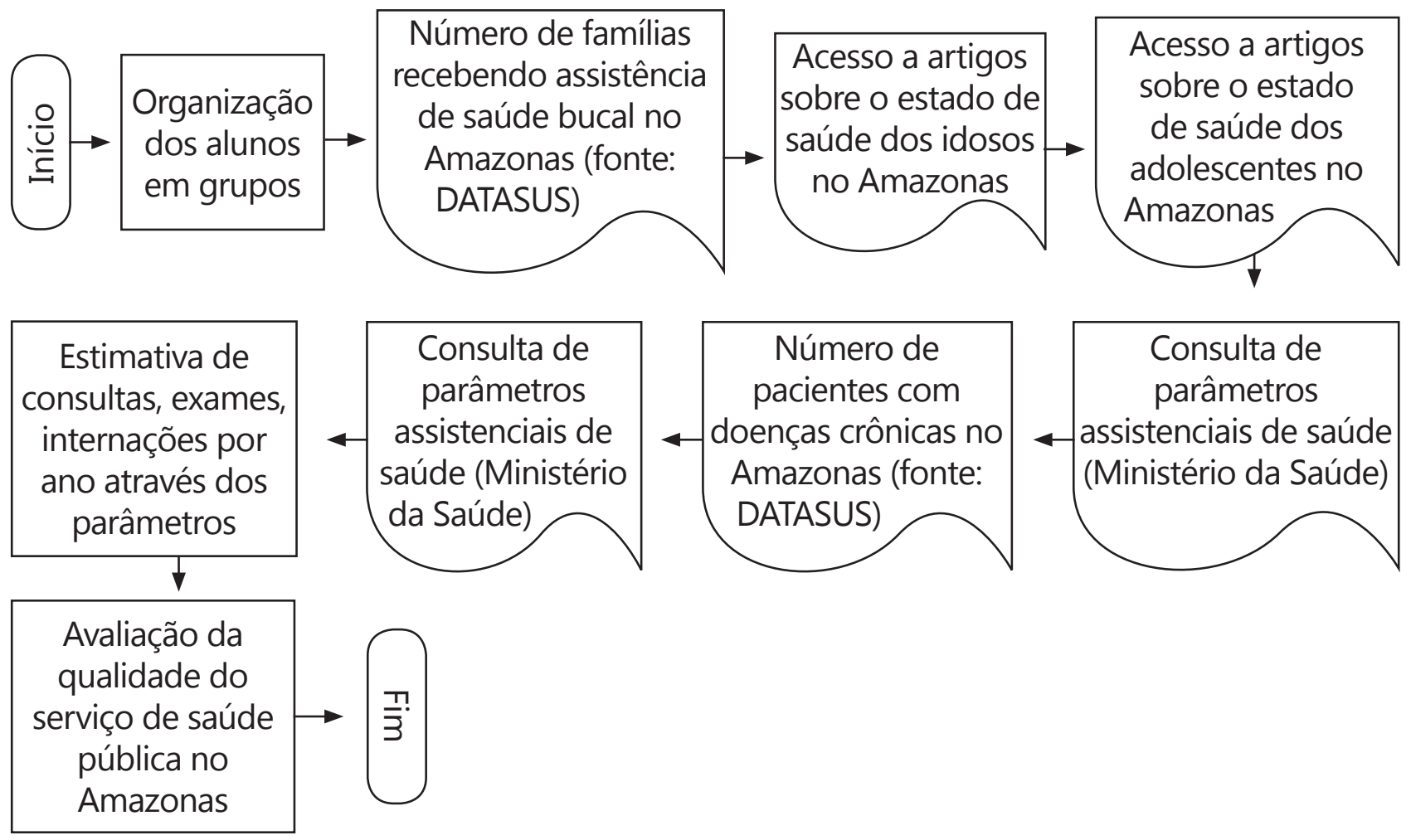

Figura 2 - Fluxograma da oficina II de planejamento. Elaborado por Rafael Esdras.

foram também utilizados os dados disponibilizados do DATASUS.

A última oficina de planejamento se voltou para a problemática atual enfrentada pela cidade de Manaus com o fluxo migratório intenso de povos indígenas venezuelanos. Inicialmente, realizou-se um brainstorming, a qual procurou extrair o máximo possível de propostas sugeridas pelos alunos a fim de encontrar uma resolução ao problema. Posteriormente, os alunos se reuniram em grupos, organizando assim as ideias em três etapas e, em cada uma delas, respondeu-se a sete perguntas: o que; quando; por quê; como; onde; quem; e quanto. No quadro 1 , pode-se verificar o resultado da atividade. 


\section{PLANO DE AÇÃO PARA IMIGRANTES VENEZUELANOS EM MANAUS}

Objetivo: Garantir os direitos humanos dos imigrantes em vulnerabilidade social; diminuir o impacto social e biológico da imigração, oferecer soluções para contenção da imigração

\begin{tabular}{|c|c|c|c|c|c|c|c|}
\hline & O que? & Quando? & Por quê? & Como? & Onde? & Quem? & Quanto? \\
\hline$\frac{\check{I}}{\frac{\pi}{5}}$ & $\begin{array}{l}\text { Descrever } \\
\text { o perfil dos } \\
\text { imigrantes }\end{array}$ & 15/12/2017 & $\begin{array}{l}\text { Entender a } \\
\text { situação social, } \\
\text { o motivo pelo } \\
\text { qual imigraram, } \\
\text { onde estão } \\
\text { abrigados, } \\
\text { origem, modo } \\
\text { de vida, habi- } \\
\text { lidades profis- } \\
\text { sionais com as } \\
\text { quais possam } \\
\text { ganhar dinheiro }\end{array}$ & $\begin{array}{l}\text { - Softwares } \\
\text { de base de } \\
\text { dados. Ex. Epi } \\
\text { info. } \\
\text { - Formulários } \\
\text { físico }\end{array}$ & $\begin{array}{l}\text { Três principais } \\
\text { aglomerados: } \\
\text { rodoviária de } \\
\text { Manaus, Feira } \\
\text { da Panair e } \\
\text { Manaus Mo- } \\
\text { derna }\end{array}$ & $\begin{array}{l}\text { Equipe mul- } \\
\text { tidisciplinar: } \\
\text { tradutor, as- } \\
\text { sistente social, } \\
\text { secretário } \\
\text { municipal de } \\
\text { saúde, antro- } \\
\text { pólogo }\end{array}$ & $R \$ 200,00$ \\
\hline$\frac{\pi}{\frac{1}{\alpha}}$ & $\begin{array}{l}\text { Cadastrar } \\
\text { as famílias } \\
\text { imigrantes }\end{array}$ & 16/02/2017 & $\begin{array}{l}\text { Fazer controle; } \\
\text { descobrir o } \\
\text { perfil epidemio- } \\
\text { lógico deles, } \\
\text { quantificar para } \\
\text { direcionar as } \\
\text { ações }\end{array}$ & $\begin{array}{l}\text { - Formulários } \\
\text { físicos } \\
\text { - Software } \\
\text { de base de } \\
\text { dados }\end{array}$ & $\begin{array}{l}\text { Três pontos } \\
\text { de cadastro: } \\
\text { rodoviária de } \\
\text { Manaus, Feira } \\
\text { da Panair e } \\
\text { Manaus Mo- } \\
\text { derna }\end{array}$ & $\begin{array}{l}\text { Equipe mul- } \\
\text { tidisciplinar: } \\
\text { tradutor, as- } \\
\text { sistente social, } \\
\text { secretário } \\
\text { municipal de } \\
\text { saúde, antro- } \\
\text { pólogo }\end{array}$ & $R \$ 200,00$ \\
\hline 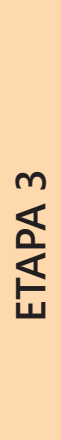 & $\begin{array}{l}\text { Fazer rastreio } \\
\text { de doenças }\end{array}$ & 24/02/2017 & $\begin{array}{l}\text { Estabelecer me- } \\
\text { didas preventi- } \\
\text { vas e curativas; } \\
\text { identificar } \\
\text { endemias da } \\
\text { região de ori- } \\
\text { gem, evitando } \\
\text { contaminação } \\
\text { dos moradores } \\
\text { locais }\end{array}$ & $\begin{array}{l}\text { Triagem. } \\
\text { Exame clínico, } \\
\text { exames labo- } \\
\text { ratoriais }\end{array}$ & $\begin{array}{l}\text { Levar ambula- } \\
\text { tórios de rua } \\
\text { para o local } \\
\text { onde estão }\end{array}$ & $\begin{array}{l}\text { Equipe } \\
\text { multidiscipli- } \\
\text { nar: tradutor, } \\
\text { profissionais } \\
\text { de saúde da } \\
\text { equipe do } \\
\text { ambulatório } \\
\text { de rua }\end{array}$ & $R \$ 200,00$ \\
\hline
\end{tabular}

Quadro 1 - Resultado da última oficina de planejamento na Saúde Coletiva IV. Elaborado por Rafael Esdras.

\section{Discussão}

É fundamental para um médico em formação ser ensinado a se inserir na discussão de propostas de políticas de saúde. Como relatado por Rios e Sirino (2015), para ser um profissional da área há não somente uma necessidade do conhecimento científico e tecnológico, é preciso também um envolvimento com conhecimento de natureza humanística e social no sentido de avaliar políticas e conduzir sistemas e serviços de saúde.

A partir das experiências vivenciadas na disciplina Saúde Coletiva IV, foi possível verificar a importância de trabalhar em grupo compartilhando diferentes pontos de vistas sobre o funcionamento do serviço público de saúde no Amazonas. Além disso, a interdisciplinaridade complementou as 
aulas teóricas e práticas, houve a participação de uma mestranda da área de Geografia da Saúde. Por meio da sua participação, obteve-se conhecimento sobre instrumentos úteis para vigilância epidemiológica, como o uso do geoprocessamento e sistemas de informação em saúde.

Desse modo, ao final, alguns alunos ficaram prontos para produzir trabalhos científicos e projetos de pesquisa, colocando em prática todos os conhecimentos adquiridos. Portanto, considerando a falta de abordagem adequada do tema no ensino observada por Teixeira e Sá (1996), tal relato serve de incentivo para que atividades semelhantes a essas possam ser incluídas na formação de futuros médicos no Brasil.

\section{Referências Bibliográficas}

BRASIL. Ministério da Saúde. Sistema de Informação de Atenção Básica. Rio de Janeiro: Departamento de Informação do SUS (DATASUS). Disponível em: <http://www2.datasus.gov.br/SIAB/ index.php >. Acessado em 28 fev. 2018.

BRASIL. Ministério da Saúde. "Portaria N. 1.101, de 12 de junho de 2002. Parâmetros Assistenciais do SUS". Disponível em: <sna.saude.gov.br/legisla/legisla/informes/GM_P1.101_02informes.doc $>$. Acessado em 28 fev. 2018.

CARVALHO, Yara Maria de \& CECCIM, Ricardo Burg. "Formação e Educação em Saúde: Aprendizados com a Saúde Coletiva". In: CAMPOS, G. W. S.; MINAYO, M. C. S.; AKERMAN, M.; DRUMOND JR., M. \& CARVALHO, Y. M. (orgs.). Tratado de Saúde Coletiva. São Paulo: Hucitec; Rio de Janeiro: Fiocruz; 2006, pp. 149-82.

INSTITUTO BRASILEIRO DE GEOGRAFIA E ESTATÍSTICA. Estimativas de População. 2017. Disponível em: <https://www.ibge.gov.br/estatisticas-novoportal/ sociais/populacao >. Acessado em 28 fev. 2018.

PAIM, Jairnilson Silva. "Epidemiologia e Planejamento: a Recomposição das Práticas Epidemiológicas na Gestão do SUS". Ciência \& Saúde Coletiva, vol. 8, n. 2, 2003, pp. 557-67.

"Planejamento em Saúde para não Especialistas". In: CAMPOS, G. W. S.; MINAYO, M. C. S.; AKERMAN, M.; DRUMOND JR., M. \& CARVALHO, Y. M. (orgs.). Tratado de Saúde Coletiva. São Paulo: Hucitec; Rio de Janeiro: Fiocruz; 2006, pp. 767-782.

RIOS, Izabel Cristina \& SIRINO, Caroline Braga. "A Humanização no Ensino de Graduação em Medicina: o Olhar dos Estudantes". Revista Brasileira de Educação Médica, vol. 39, n. 3, 2015, pp. 401-409.

RODRIGUES, Patrícia da Rocha \& DAVID, Helena Maria Scherlowski Leal. "Determinação ou Determinantes? Uma Discussão com Base na Teoria da Produção Social da Saúde". Rev. Esc. Enferm USP, vol. 49, n. 1, 2015, pp. 129-135.

SOUZA, Káren Mendes Jorge de; SEIXAS, Clarissa Terenzi; DAVID, Helena Maria Scherlowski Leal David \& COSTA, Aline Queiroz da. "Contribuições da Saúde Coletiva para o Trabalho de Enfermeiros". Revista Brasileira de Enfermagem, vol. 70, n. 3, 2017, pp. 569-576.

TAMBELlini, A. T \& CÂMARA, V. M. "A Temática Saúde e Ambiente no Processo de Desenvolvimento do Campo da Saúde Coletiva: Aspectos Históricos, Conceituais e Metodológicos". Ciênc. Saúde Colet., vol. 3, n. 2, 1998, pp. 47-59.

TEIXEIRA, Carmem Fontes \& SÁ, Marilene de Castilho. "Planejamento \& Gestão em Saúde: Situação Atual e Perspectivas para a Pesquisa, o Ensino e a Cooperação Técnica na Área". Ciênc. Saúde Colet., vol. 1, n. 1, 1996, pp. 80-103. 